\title{
Screening for Diabetic Retinopathy with a Nonmydriatic Ultra-Wide-Field Retina Camera by Family Medicine Physicians
}

\author{
Bryan A. Farford, DO, Abhimanyu S. Abuja, BS, Michael W. Stewart, MD, \\ James M. Naessens, ScD, and Joshua J. Keith, DO
}

Purpose: Diabetic retinopathy (DR) is the leading cause of blindness among working-aged adults aged 20 to $\mathbf{7 4}$ years. Despite professional association guidelines that recommend yearly screening for DR, only about $60 \%$ of Americans with diabetes mellitus (DM) receive annual examinations. The purpose of this 2-phase study was to determine the ability of family medicine (FM) physicians to accurately interpret retinal images of patients with DM.

Methods: Five FM physicians received a 1-hour lecture on DR by a retinal specialist after which the physicians were shown 30 ultrawide-field retina images and asked to determine whether the images contained signs of DR (phase 1).

Patients: Patients with DM who had not received an eye examination within the past year underwent nonmydriatic retinal photography in a FM clinic (phase 2). The 5 FM physicians were asked to evaluate the images for signs of DR and the images were simultaneously sent to a retinal specialist for independent interpretation. The diagnoses of the FM physicians and retina specialist were compared. Patients were informed of their results and were asked to complete a brief telephone survey regarding their experience with the screening process.

Results: Thirty retina images, 5 with DR and 25 without DR, were included in the postlecture assessment. Each of the 30 images was reviewed by all 5 FM physicians. Of the 5 images with DR, 3 were correctly diagnosed by all 5 FM physicians, 1 was correctly diagnosed by 4 , and 1 was accurately

diagnosed by 3 . Overall accuracy for the 5 FM physicians was $100 \%, 100 \%, 100 \%, 97 \%$, and $87 \%$.

Among the 34 patients included in phase $2,3(8 \%)$ were diagnosed with DR by the retinal specialist but $8(24 \%)$ were diagnosed with DR by the FM physicians. Of the 3 patients with DR confirmed by the retinal specialist, only 1 was detected by the FM physicians (sensitivity, 33\%; 95\% CI, 1\% to 91\%). of the 31 patients without DR as determined by the retinal specialist, 24 were accurately diagnosed by the FM physicians (specificity, $77 \%$; $95 \%$ CI, 59\% to 90\%). The screening procedure was considered easy/efficient by 28 of $31(90 \%)$ respondents.

Conclusion: To improve early detection of DR new screening methods should be considered. FM physicians were able to accurately identify DR on postlecture images but were not as accurate when evaluating images taken from patients in the FM clinic. Patients found the screening process to be easy and efficient. This study was limited by the small sample size, particularly the limited number of DR cases. Future studies that include cases with a wide variation of DR severity are needed to determine the accuracy of FM physicians at detecting DR in a clinical setting. ( $\mathrm{J}$ Am Board Fam Med 2021;34:231-237.)

Keywords: Blindness, Diabetic Retinopathy, Diabetes Mellitus, Family Medicine, Obesity, Retina, Screening

\section{Introduction}

Diabetic retinopathy (DR) is the leading cause of blindness among working adults between the ages

This article was externally peer reviewed.

Submitted 20 June 2020; revised 18 September 2020; accepted 20 September 2020.

From the Department of Family Medicine, Mayo ClinicFlorida, Jacksonville, FL (BAF, JJK); Charles E. Schmidt College of Medicine, Florida Atlantic University, Boca Raton, FL (ASA); Department of Ophthalmology, Mayo Clinic-Florida, Jacksonville, FL (MWS); Health Care Policy of 20 and 74 years. ${ }^{1}$ Among Americans with type 2 diabetes mellitus (DM), approximately $40 \%$ have $\mathrm{DR}$ and this number jumps to $86 \%$ among patients

and Research, Mayo Clinic-Rochester, Rochester, MN (JMN).

Funding: Intramural funding from Mayo Clinic-Florida was provided to support this research.

Conflict of interest: None.

Corresponding author: Bryan A Farford, DO, Department of Family Medicine, Mayo Clinic-Florida, 742 Marsh Landing Parkway, Jacksonville, FL 32250 (E-mail: farford.bryan@mayo). 
with type $1 \mathrm{DM}^{2}$ Screening is an important component of preventative care since patients can be asymptomatic and still have vision-threatening DR. Early detection of DR determines the need for referral when treatment can be performed before vision loss becomes permanent. ${ }^{3-5}$ Several professional organizations, including the American Diabetes Association and the American Academy of Ophthalmology, have developed DR screening guidelines, but only $60 \%$ of Americans with DM are in compliance. ${ }^{4}$

In the primary care setting, DR screening typically requires that a separate appointment be made with an eye care provider, a time-consuming process that may be challenging for many patients. Barriers to DR screening include poor access to ophthalmic care, time constraints, out-of-pocket expenses, insufficient patient knowledge about DR, and a lack of care coordination, ${ }^{6}$ which disproportionately affect low-income and minority patients. ${ }^{6}$ The DR screening rate among minority patients was only $49 \%$ in $2009,{ }^{6}$ and even in urban areas with sufficient access to specialized eye care, annual DR screening appointments are frequently missed due to lack of awareness, difficulty in booking appointments, and long wait times. ${ }^{7}$

Retinal imaging with a nonmydriatic, ultrawidefield fundus camera is an efficient and cost-effective method of screening patients for DR. ${ }^{8,9}$ Effective DR screening has been performed in the primary care setting with the acquisition of retinal images followed by tele-ophthalmology consultation. ${ }^{10}$ But tele-ophthalmology-based review of images increases cost and prolongs screening time, and if primary care physicians were able to accurately evaluate retinal images, access to DR screening could be markedly increased. The primary purpose of this study was to determine how accurately family medicine (FM) physicians could interpret retinal images of patients with $\mathrm{DM}$, and a secondary goal was to assess patient satisfaction with the screening process.

\section{Methods}

This 3 phase study was approved by the Mayo Clinic Institutional Review Board. Eligible patients provided informed written consent before being enrolled.

\section{Phase 1}

Five board-certified FM physicians received a 1-hour lecture on DR by a retinal specialist. These physicians were shown several images of normal retinas as well as varying stages of DR. Following the lecture, the physicians were shown 30 retinal images that had previously been taken in an ophthalmology clinic and they were asked to identify whether or not the images contained DR. The physicians were encouraged to use an image of a normal retina to assist with their decision making.

\section{Phase 2}

Thirty-four patients were recruited from a FM clinic in Northeast Florida and consented to participate in the study. Inclusion criteria included a diagnosis of DM (type 1 or 2), 18 years of age or older, and no history of DR. Exclusion criteria included history of an eye examination performed within the previous 12 months or known retinal pathology. Of the 34 patients, some were randomly selected using a report that was generated through the electronic health record and others were identified during routine clinic visits. The ages of the patients ranged from 30 to 89 years with an average age of 62 years.

Before performance of the study, $2 \mathrm{FM}$ registered nurses (RNs) were trained by a Certified Ophthalmic Assistant (COA) to use an Optos 200Tx (Optos, Inc., Marlborough, MA), a nonmydriatic, ultrawide-field retina camera. The nurses demonstrated competencies by performing the necessary steps to capture retinal images and the Certified Ophthalmic Assistant monitored and assisted the RNs during image acquisition from the first 5 patient enrolled in the study.

The study was conducted from June 1, 2019 to December 1, 2019. During this period, 34 patients underwent bilateral retinal photography by the trained RNs in the FM clinic. Image acquisition took approximately 5 minutes for each patient, all the images taken during the study were of good quality, and no patient needed to return for additional images. To make this service convenient for the patients, images were often acquired when the patients were scheduled to be in the clinic for other services such as lab work or physician visits.

The images were uploaded into the electronic medical record. One of the FM physicians was notified that the photographs were ready for review and the images were simultaneously sent to the retinal specialist through secured e-mail for interpretation. The physicians and the retina specialist independently responded to the $\mathrm{RN}$ indicating if the images had signs of DR. No patient had underlying pathology, including cataracts, that impeded the interpretation. 


\section{Phase 3}

Patients were notified of the screening results by telephone and a 2-question survey was performed. Patients were asked the following: 1) Was the screening process an easy/efficient way to be screened for diabetic-related eye disease?; 2) What is the most they would be willing to spend out-of-pocket for this service if it was not covered by their insurance?

\section{Results}

\section{Phase 1}

Thirty retina images, 5 with DR and 25 without $\mathrm{DR}$, were reviewed by each of the 5 participating FM physicians. Of the 5 images with DR, 3 were accurately diagnosed by all 5 FM physicians, 1 was accurately diagnosed by 4 of the 5 FM physicians, and 1 was accurately diagnosed by 3 of the 5 FM physicians. Overall diagnostic accuracy for the 5 physicians was $100 \%, 100 \%, 100 \%, 97 \%$, and $87 \%$ (Appendix Table 1).

\section{Phase 2}

Fundus photographs were obtained from 34 patients in the FM clinic. From these photographs, the retina specialist diagnosed DR in $3(8 \%)$ patients whereas the FM physicians diagnosed DR in 8 (24\%) patients. Of the 3 patients diagnosed with DR by the retinal specialist, only 1 was detected by the FM physicians (sensitivity, 33\%; 95\% CI, 1\% to $91 \%$ ). Of the 31 patients diagnosed by the retinal specialist to not have DR, 24 were diagnosed by the FM physicians to not have DR (specificity, $77 \%$; $95 \%$ CI, $59 \%$ to $90 \%)$. Other measures of diagnostic accuracy are included in Table 1.

\section{Phase 3}

Patients completed a 2-question survey to assess their satisfaction with the screening process and to determine how much they would be willing to pay out-of-pocket for the screening. The screening procedure was considered easy/efficient by 28 of 31 (90\%) respondents. Twenty (64\%) of respondents were willing to pay at least $\$ 50$ for this screening with the most frequently named price (14 of 30; $47 \%$ ) also being $\$ 50$ (see Table 2).

\section{Discussion}

This 3-phase study showed that FM physicians were able to identify DR in preselected photographs with an average accuracy of $97 \%$. Because physicians viewed all 30 retinal images shortly after the DR lecture and were able to compare the different images to each other before committing diagnoses, their accuracy rates may have been bolstered. But when the same physicians were asked to evaluate photographs taken from a series of patients with a nonmydriatic, ultrawide-field retina camera in the FM clinic, sensitivity (33\%) and specificity (77\%) were diminished.

Poor diagnostic accuracy may adversely affect patients with DM. Overdiagnosis of DR by FM physicians (low specificity) may lead to unnecessary referrals to ophthalmology, unwarranted costs, and decreased patient satisfaction. Missed diagnoses of moderate or severe DR (low sensitivity) might delay the initiation of treatment and result in permanent vision loss. If mild background DR is missed, however, vision loss may occur.

The third phase of the study provided insight into the convenience and value of providing DR screening in a primary care setting. Since $90 \%$ of

Table 1. Initial Estimates of Diagnostic Accuracy of Interpretation of Retinal Images of 34 Patients by Family Physicians

\begin{tabular}{|c|c|c|c|c|c|c|}
\hline & DR Finding & Presumed DR & No DR & Total & Fraction (\%) & $95 \%$ CI, \% \\
\hline Retinal specialist results & 3 & - & 31 & 34 & $3 / 34(8)$ & $(2-24)$ \\
\hline Family physician results & 1 & 7 & 26 & 34 & $8 / 34(24)$ & $(11-41)$ \\
\hline Sensitivity & & & & & $1 / 3(33)$ & $(1-91)$ \\
\hline Specificity & & & & & $24 / 31(77)$ & $(59-90)$ \\
\hline PPV & & & & & $1 / 8(13)$ & $(0-53)$ \\
\hline NPV & & & & & $24 / 26(92)$ & $(75-99)$ \\
\hline Accuracy & & & & & $25 / 34(74)$ & $(56-87)$ \\
\hline
\end{tabular}

DR, diabetic retinopathy; NPV, negative predictive value; PPV, positive predictive value; CI, confidence interval. 
Table 2. Patient Follow-Up Survey

\begin{tabular}{lcc}
\hline Question & $\begin{array}{c}\text { No. of } \\
\text { Responses }\end{array}$ & $\mathrm{N}(\%)$ \\
\hline Did you find the retinal imaging an easy/ & 31 & \\
$\quad$ efficient way of screening for eye & & \\
$\quad$ disease as a result of having diabetes? & & $28(90)$ \\
Yes & $1(3)$ \\
No & $2(7)$ \\
Unknown & \\
If you had to pay for retinal imaging out- & 30 & \\
of-pocket what is the maximum & \\
$\quad$ amount you would be willing to pay? & \\
$\$ 25$ & $7(23)$ \\
$\$ 50$ & $14(47)$ \\
$\$ 75$ & $3(10)$ \\
$\$ 100$ & $2(7)$ \\
Unknown & $4(13)$ \\
\hline
\end{tabular}

patients found this process to be easy and efficient, obtaining photographs in the FM clinic could improve the screening rates for DR even if there are out-of-pocket expenses. Prior studies have noted a lack of time in the patient's schedule and long wait times for appointments with an ophthalmologist as reasons for the low yearly screening rate. ${ }^{11}$ This study found that retinal imaging when the patient was already scheduled to be in the FM clinic was convenient and appreciated.

Many commercial and government insurances cover retinal imaging and interpretation but several studies have highlighted financial concerns, such as the cost of an eye examination and the lack of insurance, as key factors why patients with DM may forgo routine eye exams. ${ }^{12}$ This financial burden may be an important barrier to eye care screening, especially for those without adequate health insurance. The yearly cost to the federal government for each person below the age of 65 years who develops blindness is $\$ 14,296$, whereas the per patient cost of DR screening by a primary care physician (PCP) was just $\$ 31 .{ }^{13}$ Many patients in this study indicated that they would be willing to pay $\$ 50$ for DR screening in a primary care office suggesting that low-cost DR screening in the primary care setting may increase the number of patients screened each year and assist in reducing the financial burden of vision loss and blindness.

Training RNs to perform retinal photography proved cost effective since no additional staff was needed to offer this service line. The efficiency of the screening did not add significantly to the daily responsibilities of the $\mathrm{RNs}$ and it was noted that they took the opportunity to educate patients on DR and the importance of routine screening.

The physicians were not sufficiently accurate with their diagnoses to justify implementing this process into daily practice but the value of this service warrants additional work to make this a new standard for care. We believe that improvements could be made to the training provided in phase 1 of the study. The FM physicians received a 1-hour lecture on DR by a retinal specialist and though the lecture provided an introduction to the interpretation of retinal images, the training could have offered real-time image assessment with direct feedback from the retinal specialist. In addition, the FM physicians were not provided results of the postlecture image assessment, which could have been done directly with the retinal specialist to explain inaccurate readings. Future studies to determine whether expanded training could improve the accuracy of DR screening by FM physicians are warranted.

The number of participating patients in phase 2 of this study was small, which limited the number of images available for physician evaluation. This may have hindered their ability to differentiate a normal retina from 1 with early DR. As in phase 1, the FM physicians did not receive the results or feedback on their image interpretation, a strategy that could have improved accuracy as the study progressed. A longer study with more participants and timely feedback to the physicians reviewing the images may improve accuracy.

Many of the images shown in phase 1 contained moderate to advanced DR that may have been obvious to the family physicians. Conversely, the very early stages of DR identified by the retinal specialist in phase 2 may not have been obvious to the FM physicians. Had the findings in Phase 2 been more advanced, the FM physicians may have diagnosed more accurate.

One emerging area of research that could improve the accuracy of image interpretation in the primary care setting is artificial intelligence (AI). An AI-based system analyzed color fundus images taken in the primary care clinic in Australia and assigned a binary DR or no DR grade. This information helped the primary care physician (PCP) determine the need for referral to an ophthalmologist. ${ }^{14}$ The AI-based system identified all 10 patients with clinically significant (2) and mild (8) 
DR and had a specificity of $92 \%$, but produced several false positives. ${ }^{14}$

Other reasons support imaging of DM patients in the primary care setting. Previous epidemiologic studies found substantial socioeconomic disparities in diabetes care ${ }^{15}$ and even among patients receiving diabetes education, those with lower monthly income and a lower education level are less likely to be screened for DR. ${ }^{15}$ Lower socioeconomic status has consistently been correlated with a higher prevalence of DR and visual impairment. ${ }^{16}$ Retinal imaging at the time of diabetes care could mitigate the differences in screening rates.

Previous studies reported higher prevalence rates, lower screening rates, and undertreatment of DR in certain minority populations. ${ }^{13,18} \mathrm{DR}$ is responsible for $17 \%$ of vision loss in African Americans (vs $8 \%$ in non-Hispanic whites) ${ }^{13,17}$ and the prevalence of DR in some Native American tribes is $45.3 \%{ }^{13}$ Acquiring DR images with a nonmydriatic retinal camera in the primary care setting could improve screening for vulnerable populations.

Diabetic screening in the primary care setting may reduce the number of referrals to ophthalmology. ${ }^{13}$ Fewer DM referrals would improve access to retinal specialists for patients with established retinopathy and would enable ophthalmologists to devote more time and resources to treating patients with established disease. ${ }^{13}$ Earlier evaluations and treatment would reduce the incidence of vision loss.

Limitations of this study include small sample size and limited number of patients with DR. This created wide CIs, which question the rate of diagnostic accuracy. Larger studies with more patients and physicians are needed to better understand the ability of FM physicians to use retina cameras for DR screening. The amount that patients are willing to pay for the convenience of this service may not be representative of a larger patient population. The participants in this study were from a suburban area in Northeast Florida and although their responses provide insight into the value they place on the service, it may be valued higher or lower by individuals in rural or urban settings.

\section{Conclusion}

DR screening by FM physicians in the primary care clinic offers several benefits. Patients find the service to be convenient and efficient, which should improve screening rates, increase early detection, and allow for timely intervention to prevent vision loss. Nurses and other staff members can be trained to capture the images, thereby avoiding the need to add additional staff for this service, and they can help educate patients about DR and the importance of routine screening.

Direct and indirect costs attributed to DR are a major financial burden on the US health care system especially since lower socioeconomic groups and ethnic populations are disproportionally affected. Costeffective screening in the FM clinic can help lessen the health care disparities experienced by individuals within these groups.

FM physicians should be appropriately trained to provide real-time interpretation of retinal images, though this study suggests that a more robust training program may be required. Newer technologies, such as AI, may improve the diagnostic accuracy in the FM clinic but further studies are needed. Because of the rapidly increasing number of patients with DM, DR will remain a major health care issue for decades, thereby emphasizing the need for innovative practice models.

To see this article online, please go to: http://jabfm.org/content/ 34/1/231.full.

\section{References}

1. Lee R, Wong TY, Sabanayagam C. Epidemiology of diabetic retinopathy, diabetic macular edema and related vision loss. Eye Vis (Lond) 2015;2:17.

2. Cheung N, Mitchell P, Wong TY. Diabetic retinopathy. Lancet 2010;376:124-36.

3. Vujosevic S, Aldington SJ, Silva P, et al. Screening for diabetic retinopathy: new perspectives and challenges. Lancet Diabetes Endocrinol 2020;8: 337-47.

4. Flaxel CJ, Adelman RA, Bailey ST, et al. Diabetic Retinopathy Preferred Practice Pattern ${ }^{\circledR}$. Ophthalmology 2020;127:P66-P145.

5. American Academy of Ophthalmology Retina/Vitreous Panel. Preferred Practice Pattern ${ }^{\circledR}$ Guidelines. Diabetic retinopathy. San Francisco, CA: American Academy of Ophthalmology; 2016.

6. Lu Y, Serpas L, Genter P, Anderson B, Campa D, Ipp E. Divergent perceptions of barriers to diabetic retinopathy screening among patients and care providers, Los Angeles, California, 2014. Prev Chronic Dis 2016;13:E140.

7. Kalogeropoulos D, Kalogeropoulos C, Stefaniotou $M$, Neofytou M. The role of tele-ophthalmology in diabetic retinopathy screening. J Optom 2020;13: 262-268. 
8. Hu J, Chen R, Lu Y, et al. Single-field non-mydriatic fundus photography for diabetic retinopathy screening: a systematic review and meta-analysis. Ophthalmic Res 2019;62:61-7.

9. Kirkizlar E, Serban N, Sisson J, Swann J, Barnes C, Williams M. Evaluation of telemedicine for screening of diabetic retinopathy in the Veterans Health Administration. Ophthalmology 2013;120:2604-10.

10. Jani PD, Forbes L, Choudhury A, Preisser JS, Viera AJ, Garg S. Evaluation of diabetic retinal screening and factors for ophthalmology referral in a telemedicine network. JAMA Ophthalmol 2017;135:706-14.

11. Piyasena MMPN, Murthy GVS, Yip JLY, et al. Systematic review on barriers and enablers for access to diabetic retinopathy screening services in different income settings. PLoS One 2019;14:e0198979.

12. Graham-Rowe E, Lorencatto F, Lawrenson JG, et al. Barriers to and enablers of diabetic retinopathy screening attendance: a systematic review of published and grey literature. Diabet Med 2018;35:1308-19.

13. Barsegian A, Kotlyar B, Lee J, Salifu MO, McFarlane SI. Diabetic Retinopathy: focus on minority populations. Int $\mathrm{J}$ Clin Endocrinol Metab 2017;3:34-45.
14. Kanagasingam Y, Xiao D, Vignarajan J, Preetham A, Tay-Kearney M, Mehrotra A. Evaluation of artificial intelligence-based grading of diabetic retinopathy in primary care. JAMA Netw Open 2018;1: e182665.

15. Lee YH. Socioeconomic differences among community-dwelling diabetic adults screened for diabetic retinopathy and nephropathy: the 2015 Korean Community Health Survey. PLoS One 2018;13:e0191496.

16. Low JR, Gan ATL, Fenwick EK, et al. Poor person and areal socioeconomic status is associated with increased diabetic retinopathy and visual impairment incidence and progression. Br J Ophthalmol 2020; doi: http://dx.doi.org/10.1136/bjophthalmol2020-316430.

17. Muñoz B, West SK, Rubin GS, et al. Causes of blindness and visual impairment in a population of older Americans: The Salisbury Eye Evaluation Study. Arch Ophthalmol 2000;118:819-25.

18. Kirk JK, Passmore LV, Bell RA, et al. Disparities in A1C levels between Hispanic and Non-Hispanic white adults with diabetes: a meta-analysis. Diabetes Care 2008;31:240-6. 
Appendix 1.

Appendix Supplementary Table 1 . Accuracy of phase 1 and phase 2 results by physician

\begin{tabular}{lllll}
\hline & \multicolumn{2}{c}{ Phase 1 Accuracy } & \multicolumn{2}{c}{ Phase 2 Accuracy } \\
\cline { 2 - 5 } Family Physician & Fraction (\%) & $95 \%$ CI & Fraction (\%) & $95 \%$ CI \\
\hline 1 & $29 / 30(97)$ & $(83,99)$ & $7 / 7(100)$ & $(59,100)$ \\
2 & $30 / 30(100)$ & $(88,100)$ & $5 / 7(71)$ & $(29,96)$ \\
3 & $30 / 30(100)$ & $(88,100)$ & $5 / 7(71)$ & $(29,96)$ \\
4 & $26 / 30(87)$ & $(69,96)$ & $3 / 6(50)$ & $(12,88)$ \\
5 & $30 / 30(100)$ & $(88,100)$ & $5 / 7(71)$ & $(29,96)$ \\
\hline Overall & $145 / 150(97)$ & $(92,99)$ & $25 / 34(74)$ & $(56,87)$ \\
\hline
\end{tabular}

